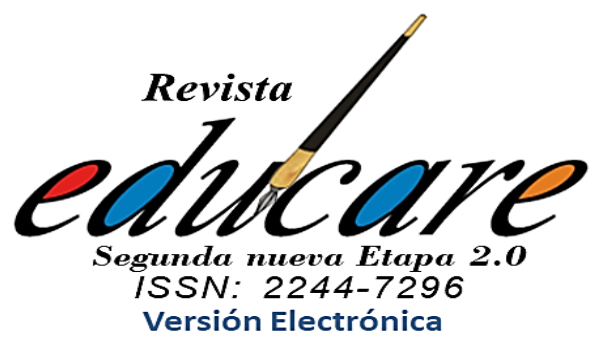

Volumen 25 No. 2 Mayo - Agosto 2021

(306-321)

Jannet del Rocío Cóndor Chicaiza*

ORCIID: https://orcid.org/0000-0001-6209-4135

Achik Ninari Chimba Santillán**

ORCIID: https://orcid.org/0000-0001-9720-2458

María Gladys Cóndor Chicaiza****

ORCID: https://orcid.org/0000-0001-6500-3454

Milton Fernando Romero Obando*****

ORCIID: https://orcid.org/0000-0003-0770-2171

Richar Jacobo Posso Pacheco******

ORCIID: https://orcid.org/0000-0003-1279-9852

Ministerio de Educación (Ecuador)

Universidad Técnica de Cotopaxi

Universidad Central del Ecuador

(Ecuador)

Magister, Docente Unidad Educativa Antonio José De Sucre Email: gladyscondor6@gmail.com

Licenciada en Ciencias de la Educación, Docente CECIB Yachay Wasi, Email:sisachik@hotmail.com

*** Licenciada en Ciencias de la Educación, Docente CECIB Yachay Wasi, Email: sisachik@hotmail.com Jannet del Rocío Cóndor Chicaiza: Magister, Docente Unidad Educativa Municipal Eugenio Espejo, Email: jannetcondor19@gmail.com

**** Milton Fernando Romero Obando: Máster en artes liberales, especialización en literatura hispanoamericana, Docente Universidad Técnica de Cotopaxi, Email: milton.romero3707@utc.edu.ec

**** Ph.D. en Proyectos, Máster en Actividad Física y Salud, Máster en Nuevas Perspectivas de la Educación Personalizada en la Sociedad Digital, Docente de la Universidad Central del Ecuador, Email: rjposso@uce.edu.ec

\section{DESARROLLO DE PROYECTOS INTERDISCIPLINARIOS EN LA EDUCACIÓN REMOTA ECUATORIANA}

DEVELOPMENT OF INTERDISCIPLINARY PROJECTS IN ECUADORIAN REMOTE EDUCATION
Recibido:

15-04-2021

Aceptado:

23-07-2021 

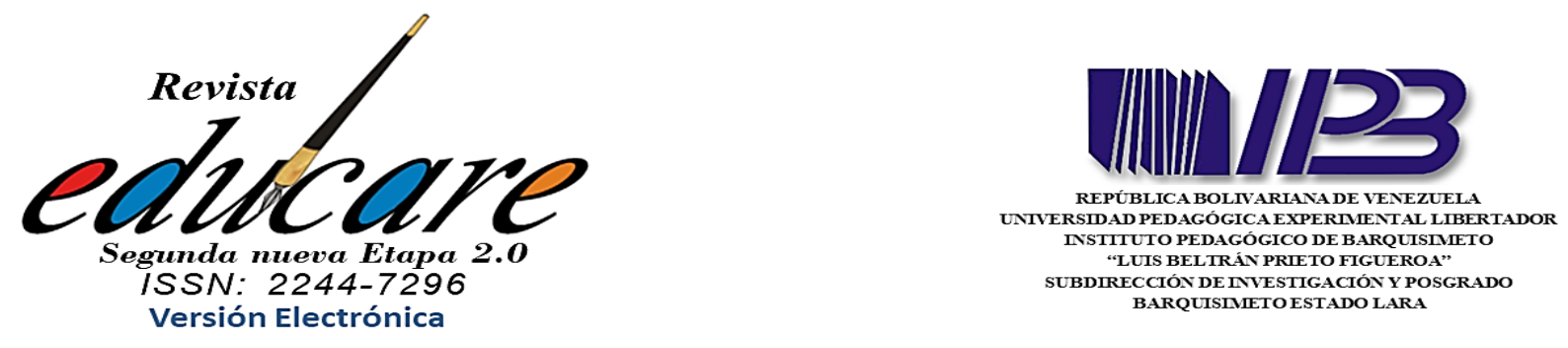

\section{Resumen}

A partir de la pandemia el currículo ecuatoriano se desarrolló a través de proyectos interdisciplinarios en el contexto de una educación remota. El objetivo de esta investigación fue determinar si los proyectos interdisciplinarios permitieron la continuidad educativa durante la pandemia. Se utilizó el paradigma cualitativo alineado a los métodos fenomenológico hermenéutico, la información se recopiló a través de entrevistas a 38 docentes de diferentes áreas del conocimiento, correspondientes a la totalidad de docentes de una institución educativa particular de la ciudad de

DESARROLLO DE PROYECTOS

INTERDISCIPLINARIOS EN LA EDUCACIÓN REMOTA ECUATORIANA

\author{
DEVELOPMENT OF \\ INTERDISCIPLINARY \\ PROJECTS IN ECUADORIAN \\ REMOTE EDUCATION
}

Quito. Los hallazgos orientaron a la conclusión de que, si bien los proyectos interdisciplinarios permitieron que los estudiantes construyan productos, no se llegó a profundizar en los aprendizajes por falta de interacción y seguimiento a los resultados de los aprendizajes, llegando solo a desarrollar los niveles de logro 1 de los indicadores de los estándares de calidad educativa en cada una de las áreas.

Palabras clave: Proyectos interdisciplinarios; aprendizajes; COVID-19; educación remota.

\section{Abstract}

Since the pandemic, the Ecuadorian curriculum was developed through interdisciplinary projects in the context of remote education. The objective of this research was to determine if interdisciplinary projects allowed educational continuity during the pandemic. The qualitative paradigm aligned to the hermeneutical phenomenological methods was used, the information was collected through interviews with 38 teachers from different areas of knowledge, corresponding to all the teachers from a particular educational institution in the city of Quito. The findings led to the conclusion that, although interdisciplinary projects allowed students to build products, learning was not deepened due to lack of interaction and monitoring of learning results, reaching only to develop achievement levels 1 of the indicators of educational quality standards in each of the areas.

Keywords: Interdisciplinary projects; learning, COVID-19; remote education. 


\section{Introducción}

El modelo educativo ecuatoriano es el constructivista social y el currículo nacional vigente 2016 se encuentra alineado en sus elementos; sin embargo, no todos los docentes lo seguían, debido a diferentes tipos de resistencia al cambio de paradigma (Posso, Barba y Otáñez, 2020). En este sentido, Minga y Ajila (2015) menciona que esto impide a que los estudiantes adquieran conocimientos de acuerdo a los ritmos y estilos de aprendizajes de cada uno, considerando también que estos docentes tampoco se alinean a las nuevas tecnologías de la información y comunicación [TIC].

La crisis pandémica ocasionada por el COVID19 puso en jaque a todos los sistemas educativos del mundo, debido a que se suspendieron las clases presenciales para evitar los contagios; en el Ecuador se asume esta realidad, y se da un alto a las actividades académicas a partir de marzo del 2020 siguiendo el llamado del decreto presidencial para el estado de excepción por la emergencia sanitaria vivida (Moreno, 2020).

No obstante, a partir de mayo del mismo año el Ministerio de Educación realiza un llamado al reinicio de actividades escolares, pero esta vez con una modalidad remota, en la que se da prioridad a las destrezas con criterio de desempeño básicas imprescindibles de cada área del conocimiento, para garantizar los aprendizajes aun dentro del contexto de la pandemia, para ello se da cabida a s un currículo priorizado que desarrollan los niveles de logro 1 de los indicadores de calidad educativa de los estándares en cada una de las áreas; manteniendo la misma estructura del currículo vigente en cuanto a ser abierto y flexible en dependencia de la necesidades y requerimientos de aprendizaje de los estudiantes (Ministerio de Educación del Ecuador, 2020)

Es de señalar que este currículo priorizado no aborda las posibilidades de aprendizaje a distancia, es decir que este integra a los estudiantes que por falta de conectividad y dispositivos electrónico no pueden tener una educación virtual; situación que da base a este currículo priorizado que permite durante el tiempo pandémico atender a esa población estudiantil no beneficiada por la conectividad. Estructuralmente el currículo priorizado se organiza por objetivos de proyecto de tipo interdisciplinarios, los cuales pueden ser construidos en proyectos contextualizados para que los estudiantes lo puedan recibir impresos en sus hogares, lo que posibilita hacer un trabajado desde la autonomía de conocimientos de los estudiantes con ayuda de los representantes legales en el que el docente se convierte en un supervisor y orientador. 
Hablar de educación remota no es hacer distinción a la educación virtual, pero si es una educación asistida por la tecnología. Desde la postura del Ministerio de Educación ecuatoriano, se trata de modalidades virtual y a distancia, en un contexto de generación de autonomía de aprendizaje de los estudiantes. La modalidad virtual en el sentido del uso y aplicación de plataformas virtuales asincrónicas y sincrónicas para la enseñanza apoyadas de metodologías activas, donde el estudiante debe disponer de conectividad y dispositivos electrónicos; y, la modalidad a distancia en la que el estudiante no cuenta con plataformas y dispositivos electrónicos para el aprendizaje, sino que cuenta con material físico entregado por las instituciones educativas, para ser desarrollados desde su autonomía con apoyo de sus familiares o personas que se encuentran a cargo de los estudiantes, (Posso et al. 2021a)

Apoyando a este enunciado Hodges et al. (2020) menciona que este término educación remota ha surgido por la crisis pandémica provocada por el COVID-19 y adoptada por varios países, siendo una estrategia de enseñanza alternativo de tipo híbrido y temporal, que garantice la adquisición de aprendizajes básicos a los estudiantes. La propuesta curricular ecuatoriana planteada para esta educación remota es de carácter interdisciplinario, alineando las destrezas con criterio de desempeño de todas las áreas disciplinarias del año o grado al cumplimiento de un objetivo de aprendizaje, evitando la segmentación del conocimiento y la elaboración de diferentes evidencias; por eso el Ministerio de educación encaminó estos objetivos a la adquisición de habilidades del siglo XXI planteadas por la UNESCO (Rodríguez et al, 2018).

Estos objetivos son desarrollados a través de proyectos interdisciplinarios como un recurso contextualizado a las necesidades y requerimientos de aprendizaje de los estudiantes, por lo que los docentes de un mismo año o grado deben reunirse para esta construcción interdisciplinar, y plantear diferentes estrategias tomando en cuenta los ritmos y estilos de aprendizaje de sus estudiantes, las posibilidades de aprender en el hogar y actividades de contención emocional, (Posso et al. 2021b)

Hasta el año lectivo 2020-2021 el Ministerio de Educación ecuatoriano planteaba los proyectos interdisciplinarios dejando a los docentes la contextualización de éstos, pero a partir del año lectivo 2021-2022 los docentes serán los encargados de construir íntegramente estos proyectos de acuerdo con las necesidades y requerimientos detectados previo diagnóstico, en correspondencia de aprendizaje del currículo priorizado y el currículo priorizado para la emergencia. 
El número de proyectos anuales para el desarrollo de las destrezas con criterio de desempeño dependerá de las necesidades conocer, por lo tanto, será decisión de los docentes de las diferentes áreas del conocimiento, además se recomienda la elaboración de dos tipos de proyectos como los humanísticos y los científicos. En todo caso los docentes serán los que decidan sobre los objetivos, los contenidos curriculares, las metodologías activas, los recursos y las formas de evaluación por proyectos interdisciplinarios.

Los proyectos tienen implícita la trasformación educativa porque invitan al estudiante a ser artífice de su proceso de aprendizajes, y cambia el rol del docente de un transmisor de la información a un mediador para la construcción del conocimiento o forma de entender la realidad. Por otra parte, garantizan la tan mencionada educación integral, porque el aprendizaje abarca las dimensiones cognitivo, social y afectivo fundamental para desarrollar las habilidades para la vida, (Cargua et al., 2019)

La calidad educativa ya no está dada por los índices de aprendizaje, sino por el reconocimiento social que tienen las instituciones educativas en la comunidad, por tanto, es deber de estas instituciones fomentar la participación activa de los estudiantes y las familias, para incluir en el proceso de aprendizaje las necesidades e intereses del desarrollo local, y que estos espacios sean verdaderos catalizadores de productivos.

En este sentido los proyectos interdisciplinarios buscan el desarrollo de las habilidades blandas como la comunicación efectiva, que invitan toda la comunidad educativa a construir y compartir el aprendizaje significativo por contexto y de lugar. Con los antecedentes antes expuestos se fundamenta el objetivo de esta investigación el cual fue determinar si los proyectos interdisciplinarios propuestos por el Ministerio de Educación ecuatoriano permitieron la continuidad educativa para la adquisición de los aprendizajes durante la pandemia.

\section{Metodología de la Investigación}

En esta investigación se utilizó el paradigma cualitativo con enfoque interpretativo abordando la perspectiva fenomenológica hermenéutica. Fenomenológica debido a que se fundamentó bajo las experiencias vividas de los docentes en el contexto de la educación remota. Hermenéutica debido a las interpretaciones del desarrollo de proyectos interdisciplinarios y la continuidad educativa, que están implícitos en el accionar y experiencias de los docentes. Se partió del supuesto 
que determina que los proyectos interdisciplinarios permiten la continuidad educativa en la pandemia, seguidamente se recogió la experiencia vivida de los docentes durante de la pandemia y se reflexionó fenomenológicamente sobre estos resultados.

La información se recopiló en el mayo del 2021, a través de entrevistas por la plataforma zoom, con 9 preguntas semiestructurada a 38 docentes de diferentes áreas del conocimiento, correspondientes a la totalidad de docentes de una institución educativa particular de la ciudad de Quito. Para este fin se diseñó un instrumento basado en tres criterios como la formación docente en proyectos interdisciplinarios, la aplicación de metodologías activas y la evaluación de los aprendizajes.

Este instrumento se validó a través de la metodología de juicio de cinco expertos, seleccionados a los criterios de Skjong y Wentworht (2000) experiencia, reconocimiento, disposición de tiempo, siendo elegidos personas con experiencia de 10 años de profesión docente, que hayan tenido publicaciones en revistas científicas, expertos en metodologías activas, dominio de proyectos interdisciplinarios e interés por participar. La validación se presentó en una sola circulación, debido a que ninguna pregunta fue excluida por tener altos valores en el coeficiente $\mathrm{V}$ de Aiken en un intervalo de confianza del $99 \%$ en los parámetros de contenido, secuencia e importancia.

\section{Tabla 1.}

Media, desviación típica, coeficiente de variación, $V$ de Aiken e intervalo de confianza en contenido

\begin{tabular}{lccccc}
\hline \multicolumn{1}{c}{ Criterio } & Pregunta & \multicolumn{5}{c}{ Contenido } \\
\hline Formación docente en & 1 & $5,00 \pm 0,00$ & 0 & V Aiken & IC (99\%) \\
proyectos & 2 & $4,90 \pm 0,31$ & 6,33 & 0,98 & $0,82-0,99$ \\
interdisciplinarios & 3 & $5,00 \pm 0,00$ & 0 & 1 & $0,86-1,00$ \\
& & & & & \\
Aplicación de & 4 & $5,00 \pm 0,00$ & 0 & 1 & $0,86-1,00$ \\
metodologías activas & 5 & $5,00 \pm 0,00$ & 0 & 1 & $0,86-1,00$ \\
& & & & & 0,80 \\
Evaluación de los & 6 & $5,00 \pm 0,00$ & 0 & 0,98 & $0,82-0,99$ \\
aprendizajes & 7 & $4,90 \pm 0,31$ & 6,33 & 0,98 & $0,82-0,99$ \\
& 8 & $4,90 \pm 0,32$ & 6,53 & 0,98 & $0,82-0,99$ \\
\hline
\end{tabular}




\section{Tabla 2}

Media, desviación típica, coeficiente de variación, $V$ de Aiken e intervalo de confianza en secuencia

\begin{tabular}{|c|c|c|c|c|c|}
\hline \multirow[t]{2}{*}{ Criterio } & \multirow[t]{2}{*}{ Pregunta } & \multicolumn{4}{|c|}{ Secuencia } \\
\hline & & Media & $\mathrm{CV}(\%)$ & V Aiken & IC $(99 \%)$ \\
\hline Formación docente en & 1 & $4,90 \pm 0,31$ & 6,33 & 0,98 & $0,82-0,99$ \\
\hline proyectos & 2 & $4,90 \pm 0,31$ & 6,33 & 0,98 & $0,82-0,99$ \\
\hline interdisciplinarios & 3 & $4,90 \pm 0,31$ & 6,33 & 0,98 & $0,82-0,99$ \\
\hline Aplicación de & 4 & $4,90 \pm 0,32$ & 6,53 & 0,98 & $0,82-0,99$ \\
\hline metodologías activas & 5 & $4,90 \pm 0,31$ & 6,33 & 0,98 & $0,82-0,99$ \\
\hline Evaluación de los & 6 & $4,90 \pm 0,31$ & 6,33 & 0,98 & $0,82-0,99$ \\
\hline aprendizajes & 7 & $4,90 \pm 0,31$ & 6,33 & 0,98 & $0,82-0,99$ \\
\hline & 8 & $4,90 \pm 0,32$ & 6,53 & 0,98 & $0,82-0,99$ \\
\hline & 9 & $4,90 \pm 0,31$ & 6,33 & 0,98 & $0,82-0,99$ \\
\hline
\end{tabular}

\section{Tabla 3}

Media, desviación típica, coeficiente de variación, $V$ de Aiken e intervalo de confianza en importancia.

\begin{tabular}{|c|c|c|c|c|c|}
\hline \multirow[t]{2}{*}{ Criterio } & \multirow[t]{2}{*}{ Pregunta } & \multicolumn{4}{|c|}{ Importancia } \\
\hline & & Media & $\mathrm{CV}(\%)$ & V Aiken & IC (99\%) \\
\hline Formación docente en & 1 & $5,00 \pm 0,00$ & 0 & 1 & $0,86-1,00$ \\
\hline proyectos & 2 & $4,90 \pm 0,31$ & 6,33 & 0,98 & $0,82-0,99$ \\
\hline interdisciplinarios & 3 & $4,90 \pm 0,31$ & 6,33 & 0,98 & $0,82-0,99$ \\
\hline Aplicación de & 4 & $5,00 \pm 0,00$ & 0 & 1 & $0,86-1,00$ \\
\hline metodologías activas & 5 & $4,90 \pm 0,32$ & 6,53 & 0,98 & $0,82-0,99$ \\
\hline Evaluación de los & 6 & $5,00 \pm 0,00$ & 0 & 1 & $0,86-1,00$ \\
\hline \multirow[t]{3}{*}{ aprendizajes } & 7 & $4,90 \pm 0,32$ & 6,53 & 0,98 & $0,82-0,99$ \\
\hline & 8 & $4,90 \pm 0,31$ & 6,33 & 0,98 & $0,82-0,99$ \\
\hline & 9 & $5,00 \pm 0,00$ & 0 & 1 & $0,86-1,00$ \\
\hline
\end{tabular}

Ninguna pregunta tuvo un coeficiente de variación superior al $20 \%$ y los valores del coeficiente V de Aiken fueron por encima del 0,98 en los parámetros de contenido, secuencia e importancia, es decir ninguna pasó a revisión. 


\section{Interpretación de los Hallazgos}

Una vez realizada las entrevistas determinadas en tres criterios: formación docente en proyectos interdisciplinarios, la aplicación de metodologías activas y la evaluación de los aprendizajes, emergieron categorías y subcategorías de cada una de ellas, a fin de determinar si los proyectos interdisciplinarios permitieron la continuidad educativa durante la pandemia.

\section{Criterio Formación docente en proyectos interdisciplinarios}

A continuación, se presenta la tabla 4 como resultado del Criterio Formación docente en proyectos interdisciplinarios, aquí se muestra e las coincidencias de opinión de los 38 docentes entrevistados, distribuidas en cuatro categorías.

\section{Tabla 4}

Resultados de la entrevista Criterio Formación docente en proyectos interdisciplinarios

\begin{tabular}{|c|c|c|c|c|}
\hline Criterios & Categorías & Subcategorías & $\begin{array}{c}\text { No. } \\
\text { respuestas }\end{array}$ & $\%$ respuestas \\
\hline \multirow{7}{*}{$\begin{array}{l}\text { Formación docente en } \\
\text { proyectos } \\
\text { interdisciplinarios }\end{array}$} & \multirow[b]{2}{*}{$\begin{array}{l}\text { Construcción de } \\
\text { proyectos } \\
\text { Interdisciplinarios }\end{array}$} & $\begin{array}{l}\text { Escasas capacitaciones en } \\
\text { educación remota y } \\
\text { construcción de proyectos } \\
\text { interdisciplinarios }\end{array}$ & 25 & 65 \\
\hline & & $\begin{array}{l}\text { Escasos conocimiento en la } \\
\text { alineación de los proyectos } \\
\text { interdisciplinarios con el } \\
\text { currículo priorizado y } \\
\text { currículo priorizado para la } \\
\text { emergencia }\end{array}$ & 27 & 70 \\
\hline & \multirow{4}{*}{$\begin{array}{l}\text { Contextualización de } \\
\text { proyectos } \\
\text { interdisciplinarios }\end{array}$} & $\begin{array}{l}\text { No se puede ajustar a la } \\
\text { realidad del estudiante debido } \\
\text { a que no se conoce }\end{array}$ & 29 & 76 \\
\hline & & $\begin{array}{l}\text { No son aplicables por la falta } \\
\text { de recursos virtuales }\end{array}$ & 21 & 55 \\
\hline & & $\begin{array}{l}\text { No se tiene conocimiento } \\
\text { sobre estrategias de contención } \\
\text { emocional }\end{array}$ & 19 & 50 \\
\hline & & $\begin{array}{l}\text { Existen diferentes realidades } \\
\text { para ajustar la } \\
\text { contextualización }\end{array}$ & 22 & 58 \\
\hline & $\begin{array}{l}\text { Trabajo interdisciplinar } \\
\text { entre las áreas del } \\
\text { conocimiento }\end{array}$ & $\begin{array}{l}\text { Existen diferentes criterios } \\
\text { entre los docentes para la } \\
\text { construcción de proyectos } \\
\text { interdisciplinarios }\end{array}$ & 21 & 55 \\
\hline
\end{tabular}




\begin{tabular}{|c|c|c|c|}
\hline & $\begin{array}{l}\text { Existe dificultades entre las } \\
\text { áreas de alinearse al objetivo } \\
\text { del proyecto interdisciplinar }\end{array}$ & 33 & 88 \\
\hline & $\begin{array}{l}\text { No se considera un tiempo } \\
\text { específico para la planificación } \\
\text { de los proyectos } \\
\text { interdisciplinarios }\end{array}$ & 32 & 83 \\
\hline \multirow{4}{*}{$\begin{array}{l}\text { Aplicabilidad en la } \\
\text { educación remota }\end{array}$} & $\begin{array}{l}\text { No todos los docentes cuentan } \\
\text { con velocidad de internet } \\
\text { adecuada }\end{array}$ & 30 & 78 \\
\hline & $\begin{array}{l}\text { Muchos estudiantes por } \\
\text { problemas de internet y } \\
\text { dispositivos no se conectan a } \\
\text { las clases sincrónicas } \\
\end{array}$ & 35 & 91 \\
\hline & $\begin{array}{l}\text { Los estudiantes tienen } \\
\text { dificultades en el trabajo por } \\
\text { proyectos }\end{array}$ & 32 & 83 \\
\hline & $\begin{array}{l}\text { Escaso apoyo en el } \\
\text { seguimiento del cumplimiento } \\
\text { de las evidencias de } \\
\text { aprendizaje por pate de los } \\
\text { familiares y representantes de } \\
\text { los estudiantes }\end{array}$ & 32 & 83 \\
\hline
\end{tabular}

Como preámbulo para el análisis de esta tabla, se puede decir que ningún país en el campo de la educación estuvo preparado para afrontar la pandemia, de la misma forma se puede decir que ningún profesional de la educación tuvo una formación alineada a una educación remota. El Ministerio de Educación ecuatoriano tuvo que ensayar con varias propuestas educativas para afrontar la pandemia, hasta plantear la construcción de proyectos interdisciplinarios en una educación remota y en el retorno progresivo a las clases presenciales.

Es por eso que se puede rescatar que la capacitación a todos los docentes de las instituciones educativas en el país fue imposible, pese a los esfuerzos propios y de los mismos docentes, y con el cambio permanente de direccionamientos en la política educativa se complicó aún más, se puede decir que las instituciones educativas de modalidad particular capacitaron mejor a sus docentes, pero las condiciones reales de los estudiantes fueron diversas, ocasionando en muchos casos que se reestructuren estrategias para que llegue el aprendizaje a los estudiantes.

El apoyo por parte de los padres de familia fue fundamental para fortalecer el aprendizaje desde los hogares, pero sin duda muchos estudiantes no tuvieron la misma suerte debido a que por las diferentes condiciones económicas, sociales, laborales y de salud provocadas por la pandemia, cada estudiante afrontó diferentes realidades como la pérdida de empleo de sus padres, la falta de dinero para alimentarse, el desalojo por impago en el arriendo, enfermedades y muerte de los 
familiares, entre otros. Se puede considerar que entre los docentes existió un interés por reinventarse y ajustarse a los nuevos requerimientos educativos que exige la educación remota, muchos docentes hicieron inversiones de capacitación y otros buscaron seguir las capacitaciones gratuitas otorgadas por el Ministerio de Educación, universidades y otras organizaciones.

Sin lugar a duda, todos los docentes cumplieron en sus funciones de formadores, pero existieron muchas circunstancias que imposibilitaron su accionar. La complejidad de alinearse a currículos que priorizaban el aprendizaje, el trabajo con proyectos interdisciplinarios, lo ajustes para llegar a acuerdos entre docentes, la falta de conectividad y de dispositivos, forjaron un espíritu de superación permanente no solo entre los docentes, sino entre toda la comunidad educativa.

Pero los más golpeados en de estructura de aprendizaje fueron los estudiantes, que por diferentes motivos no cumplieron con la atención, seriedad, disposiciones y lineamientos planteados por los docentes; considerando que un verdadero cambio de paradigma siempre iba a inestabilizar en la forma y ritmos internos de aprendizaje; aún más con la falta de movimiento y interrelación social que generaba los docentes y sus compañeros.

En la siguiente tabla se puede apreciar el criterio de formación docente en proyectos interdisciplinarios y los resultados de las entrevistas agrupadas entre categorías y subcategorías, que refleja una información importante que aporta al objetivo de esta investigación.

\section{Criterio aplicación de metodologías activas}

A continuación, se presenta la tabla 5como resultado del Criterio aplicación de metodologías activas, se muestra las coincidencias de opinión de los 38 docentes entrevistados, distribuidas en tres categorías.

\section{Tabla 5}

Resultados de la entrevista criterio aplicación de metodologías activas

\begin{tabular}{|c|c|c|c|c|}
\hline Criterios & Categorías & Subcategorías & $\begin{array}{l}\text { No. } \\
\text { respuestas }\end{array}$ & $\begin{array}{l}\% \\
\text { respuestas }\end{array}$ \\
\hline \multirow{2}{*}{$\begin{array}{l}\text { Aplicación de } \\
\text { metodologías activas }\end{array}$} & \multirow{2}{*}{$\begin{array}{l}\text { Desarrollo de la } \\
\text { autonomía estudiantil }\end{array}$} & $\begin{array}{l}\text { Los estudiantes antes de la } \\
\text { pandemia no desarrollaron la } \\
\text { autonomía del aprendizaje } \\
\end{array}$ & 33 & 88 \\
\hline & & $\begin{array}{l}\text { Durante la pandemia los estudiantes } \\
\text { desarrollaron escasamente la } \\
\text { autonomía del aprendizaje }\end{array}$ & 22 & 58 \\
\hline
\end{tabular}




\begin{tabular}{|c|c|c|c|}
\hline \multirow{4}{*}{$\begin{array}{l}\text { Motivación para el } \\
\text { aprendizaje }\end{array}$} & $\begin{array}{l}\text { Desmotivación para el aprendizaje } \\
\text { debido a situaciones económicas }\end{array}$ & 19 & 50 \\
\hline & $\begin{array}{l}\text { Desmotivación para el aprendizaje } \\
\text { debido a las condiciones de salud de } \\
\text { los familiares de los estudiantes }\end{array}$ & 21 & 55 \\
\hline & $\begin{array}{l}\text { Desmotivación para el aprendizaje } \\
\text { debido a seguimiento y } \\
\text { fortalecimiento de los familiares y } \\
\text { representantes legales }\end{array}$ & 21 & 55 \\
\hline & $\begin{array}{l}\text { Escasa aplicación de estrategias de } \\
\text { contención emocional durante las } \\
\text { clases }\end{array}$ & 19 & 50 \\
\hline \multirow{4}{*}{$\begin{array}{l}\text { Adquisición de } \\
\text { habilidades para la } \\
\text { vida }\end{array}$} & $\begin{array}{l}\text { Escaso desarrollo del trabajo de } \\
\text { equipo debido a las condiciones } \\
\text { educativas de carácter sincrónico y } \\
\text { asincrónico }\end{array}$ & 21 & 55 \\
\hline & $\begin{array}{l}\text { Los estudiantes en su gran mayoría } \\
\text { pudieron desarrollar la solución de } \\
\text { problemas para las actividades } \\
\text { planteadas en cumplimiento a los } \\
\text { objetivos de aprendizaje de los } \\
\text { proyectos interdisciplinarios }\end{array}$ & 32 & 83 \\
\hline & $\begin{array}{l}\text { Escaso desarrollo en la indagación y } \\
\text { el descubrimiento debido a las } \\
\text { condiciones de la educación remota. }\end{array}$ & 35 & 91 \\
\hline & $\begin{array}{l}\text { Escaso desarrollo del pensamiento } \\
\text { crítico y lógico }\end{array}$ & 32 & 83 \\
\hline
\end{tabular}

Escaso desarrollo de la creatividad

Para el análisis de los resultados de la aplicación de metodologías activas en los proyectos interdisciplinarios se establecieron dos líneas de comprensión, la primera antes de la pandemia como un aspecto importante que continua con la educación remota durante la pandemia. Se puede decir que en su mayoría los docentes aplicaban diferentes metodologías activas en sus clases, pero no tenían conocimiento en la variedad de metodologías activas.

Esto mismo generó que los estudiantes antes de la pandemia no desarrollaran la autonomía del aprendizaje que las metodologías activas lo construyen, adicionalmente el escaso trabajo con interacciones positivas y de retroalimentación, la experimentación de logros de aprendizaje, las posibilidades y oportunidades de aprendizaje y considerar los ritmos y estilos de aprendizaje, acrecentó que durante la pandemia se desarrolle escasamente la autonomía (Rocha, 2020).

Ya se había tomado en cuenta que esta pandemia generó muchas desgracias en la familia de los estudiantes como pérdidas de familiares, despidos, desalojos, enfermedades, mala 
alimentación, entre otras, desmotivando a los estudiantes para la continuidad educativa, pese a la aplicación de diferentes estrategias de contención emocional que direccionó el Ministerio de Educación, pero fue poco efectivo al momento de su aplicación debido a que esta realidad fue para toda la comunidad educativa.

Sin duda la aplicación de metodologías activas y de recursos como el trabajo por proyectos interdisciplinarios deben generar el desarrollo de habilidades para la vida en condiciones normales, pero aquí antecede la pandemia que por mucho que se esfuerce, siempre la falta de conectividad, dispositivos de comunicación, y condiciones en el hogar, evitará que se desarrollen estas habilidades para la vida, obteniendo básicamente el cumplimiento de las evidencias del aprendizaje basados en aprendizajes de nivel de logro 1 establecidos en los currículos priorizado y priorizado para la emergencia.

Se puede decir que frente a la pandemia los estudiantes desarrollaron otras habilidades para la vida, de acuerdo con una perspectiva de distanciamiento y aislamiento, como son las habilidades digitales y las habilidades socioemocionales, seguramente el manejo del tiempo libre y la conexión con lo acontecido con sus familiares, han generado el desarrollo de estas habilidades que propenden a una visión de sociedad que va a salir de la pandemia.

\section{Criterio la evaluación de los aprendizajes}

Se presenta la tabla 6como resultado del Criterio evaluación de los aprendizajes en los proyectos interdisciplinarios, se muestra las coincidencias de opinión de los 38 docentes entrevistados, distribuidas en tres categorías.

\section{Tabla 6}

Resultados de la entrevista Criterio la evaluación de los aprendizajes

\begin{tabular}{lllll}
\hline Criterios & Categorías & Subcategorías & $\begin{array}{l}\text { No. } \\
\text { respuestas }\end{array}$ & $\begin{array}{l}\text { \% } \\
\text { respuestas }\end{array}$ \\
\hline $\begin{array}{l}\text { La evaluación de } \\
\text { los aprendizajes }\end{array}$ & $\begin{array}{l}\text { Niveles de logro de } \\
\text { los indicadores de } \\
\text { los estándares de } \\
\text { calidad }\end{array}$ & $\begin{array}{l}\text { La mayoría de los estudiantes } \\
\text { evaluados alcanzan el nivel de } \\
\text { logro 1 de los indicadores de los } \\
\text { estándares de calidad }\end{array}$ & 33 & 88 \\
\hline
\end{tabular}




\begin{tabular}{|c|c|c|c|}
\hline & $\begin{array}{l}\text { La mayoría de los estudiantes } \\
\text { evaluados no alcanzan el nivel de } \\
\text { logro } 2 \text { de los indicadores de los } \\
\text { estándares de calidad }\end{array}$ & 32 & 83 \\
\hline \multirow{4}{*}{$\begin{array}{l}\text { Cumplimiento de } \\
\text { las evidencias de } \\
\text { aprendizajes }\end{array}$} & $\begin{array}{l}\text { Existen estudiantes que no } \\
\text { presentaron las evidencias de } \\
\text { aprendizaje dispuestos en los } \\
\text { proyectos interdisciplinarios }\end{array}$ & 22 & 58 \\
\hline & $\begin{array}{l}\text { Existen estudiantes que no } \\
\text { construyeron en su totalidad los } \\
\text { proyectos interdisciplinarios }\end{array}$ & 21 & 55 \\
\hline & $\begin{array}{l}\text { Se tuvo que aplicar diferentes } \\
\text { estrategias para la presentación de } \\
\text { evidencias de aprendizaje }\end{array}$ & 22 & 58 \\
\hline & $\begin{array}{l}\text { No se alcanzaron a retroalimentar } \\
\text { las evidencias de aprendizaje } \\
\text { presentadas }\end{array}$ & 33 & 88 \\
\hline \multirow{3}{*}{$\begin{array}{l}\text { Estrategias de } \\
\text { evaluación }\end{array}$} & $\begin{array}{l}\text { Existieron dificultades en la } \\
\text { evaluación formativa debido a las } \\
\text { posibilidades de conexión de los } \\
\text { estudiantes }\end{array}$ & 21 & 55 \\
\hline & $\begin{array}{l}\text { La comunicación directa evitó que } \\
\text { se realizada adecuadamente una } \\
\text { evaluación formativa }\end{array}$ & 33 & 88 \\
\hline & $\begin{array}{l}\text { Se evaluó menos y se valoró el } \\
\text { esfuerzo de aprendizaje. }\end{array}$ & 32 & 83 \\
\hline
\end{tabular}

Para el análisis de la tabla se puede establecer que los estragos de la pandemia se profundizaron en la educación fueron impensables, la no presencialidad ha generado brechas en los resultados del aprendizaje, debido a muchos factores anteriormente vistos. Se puede destacar que, si existe continuidad de los aprendizajes, pero alcanzando los niveles de logro 1 de los indicadores de los estándares de calidad casi en todas las áreas del conocimiento, pero se debe destacar que en un pequeño porcentaje los estudiantes no alcanzaron este nivel de logro.

Cabe señalar que principalmente esto sucedió por la escasa retroalimentación que dio el docente, ocasionado por múltiples factores como la falta de conectividad, motivación de los estudiantes, seguimiento de los padres de familia, entre otros. Otro de los aspectos a rescatar es la no presentación de evidencias de aprendizaje por parte de algunos estudiantes, ni su participación en todos los proyectos interdisciplinarios.

Muchos docentes dejaron a un lado la evaluación formativa y destacaron el esfuerzo de aprendizaje que realizaban los estudiantes. De la misma forma el Ministerio de Educación en su 
afán de la continuidad educativa, determinó que todos los estudiantes sean promovidos al nivel superior, brindando múltiples de oportunidades de evaluación y refuerzos académicos.

\section{Reflexiones Finales}

La pandemia ha demostrado que la educación es muy frágil, y que necesita del liderazgo permanente del docente para garantizar el aprendizaje, también se ha establecido que la innovación educativa debe ir a la par con la sociedad, creando nuevos entornos de aprendizaje basados en la experiencia y la investigación educativa.

$\mathrm{Si}$ bien es cierto que los proyectos interdisciplinarios permitieron que los estudiantes construyan su aprendizaje de forma activa y estimulada, estos recursos didácticos y metodológicos no lograron profundizar contenidos esenciales para la continuidad del conocimiento, debido a la falta de interacción y seguimiento a los resultados de los aprendizajes, llegando solo a desarrollar los niveles de logro 1 de los indicadores de los estándares de calidad educativa en cada una de las áreas.

Esto sumado a las políticas educativas de última hora mientras los estudiantes estaban en pleno procesos de evaluación final, en la cual anunciaban que ningún estudiante podrá ser reprobado y que todos deberán ser promovidos al inmediato superior; causando pérdida de interés en la evaluación y quizás la última oportunidad para que el estudiante pueda fortalecer algunos conocimientos de promoción.

No cabe ninguna duda que la continuidad educativa no se cortó en ningún momento durante la pandemia, que todas las estrategias metodológicas fueron adecuadas, oportunas e importantes, que se pudo enfrentar a la pandemia sin ninguna experiencia previa, que todas las acciones tomadas por las autoridades fueron asertivas, que los estudiantes reflejaron interés hacia el aprendizaje.

La organización de la comunidad educativa fue fundamental, desde casa los representantes legales y desde la escuela virtual los docentes, esta trilogía fue el éxito y el fracaso del proceso de enseñanza aprendizaje, solo resta saber qué pasará es la pos pandemia, cuáles serán los retos de la educación y cuáles serán los direccionamientos a tomar.

Será que se necesitará otra reinvención en la educación a fin de poder resolver los diferentes problemas ocasionados por la pandemia; quienes serán los llamados a apoyar estos cambios; 
seguramente otros países estarán ya preparándose para estos nuevos retos, pero lo que sí es seguro que las investigaciones de lo sucedido serán el pilar fundamental para estos cambios.

Como primer paso el diagnóstico y el refuerzo académico deben ser extendidas de acuerdo con los datos encontrados, pero no será menor a un proceso de dos meses, con lo que se asegura una planificación que retome y se fortalezca aprendizajes de grados inferiores, para apuntalar el perfil de salida del bachiller ecuatoriano, y garantizar una educación de calidad.

Lo que sí es seguro que esta pandemia permitió que los docentes se actualicen en conocimientos necesarios para su labor profesional, tomando en cuenta que el centro del aprendizaje es el estudiante, rompiendo ese paradigma tradicional; se puede decir que la pandemia dejó muchas secuelas negativas, pero en la educación permitió que los docentes se reinventaran hacia la esencia misma de lo que es ser docente y el rol que desempeña para una sociedad del conocimiento.

\section{Referencias}

Cargua, A., Posso, R., Cargua, N. y Rodríguez, A. (2019). La formación del profesorado en el proceso de innovación y cambio educativo. OLIMPIA. Revista de la Facultad de Cultura Física de la Universidad de Granma, 54(16), 140-152. https://revistas.udg.co.cu/index.php/olimpia/article/view/713

Hodges, C.B., Moore, S., Lockee, B., Trust, T., \& Bond, M.A. (2020). The Difference Between Emergency Remote Teaching and Online Learning. Educause Review. https://er.educause.edu/articles/2020/3/the-difference-between-emergency-remoteteaching-and-online-learning

Minga, O., y Ajila, B. (2015). Los modelos pedagógicos y su influencia dentro de las etapas de enseñanza-aprendizaje en el área de ciencias naturales, aplicados a los y las estudiantes de séptimo grado del subnivel de básica media, de educación general básica. [Tesis de grado. Universidad Nacional de Loja]. https://dspace.unl.edu.ec/jspui/handle/123456789/14445

Ministerio de Educación, (2020). Plan educativo aprendamos juntos en casa. Quito: Ministerio de Educación del Ecuador. https://recursos2.educacion.gob.ec/wpcontent/uploads/2020/08/LINEAM2.pdf 
Moreno, L. M. (16 de marzo de 2020). Decreto Ejecutivo No. 1017. Estado de excepción por emergencia sanitaria. Quito, Ecuador. https://www.defensa.gob.ec/wpcontent/uploads/downloads/2020/03/Decreto_presidencial_No_1017_17-Marzo-2020.pdf

Posso Pacheco, R. J., Barba Miranda, L. C., Marcillo Nacato, J. C. y Beltrán Vásquez, S. J. (2021b). Educación Física Interdisciplinaria ecuatoriana en el contexto de la covid-19. Acción, 17, 1-5. http://accion.uccfd.cu/index.php/accion/article/view/154

Posso Pacheco, R., Otáñez Enríquez, N., Cóndor Chicaiza, J., Cóndor Chicaiza, M. y Lara Chala, L. (2021a). Educación Física remota: juegos motrices e inteligencia kinestésica durante la pandemia COVID-19. PODIUM -Revista de Ciencia y Tecnología en la Cultura Física, 16(2), 564-575. https://podium.upr.edu.cu/index.php/podium/article/view/1096

Posso, R., Barba L. y Otáñez, N. (2020). El conductismo en la formación de los estudiantes universitarios. Revista Educare Segunda Nueva Etapa 2.0. 24(1), 117-133. https://doi.org/10.46498/reduipb.v24i1.1229

Rocha Espinoza, J. J. (2020). Metodologías activas, la clave para el cambio de la escuela y su aplicación en épocas de pandemia. INNOVA Research Journal, 5(3.2), 33-46. https://doi.org/10.33890/innova.v5.n3.2.2020.1514

Rodríguez Torres, Á., Posso Pacheco, R., De la Cueva Constante, R. y Barba Miranda, L. (2018). Herramientas metodológicas para el desarrollo de habilidades investigativas en los estudiantes: una praxis necesaria. Revista Científica Olimpia, 15(50), 119-132. https://dialnet.unirioja.es/descarga/articulo/6578678.pdf

Skjong, R. y Wentworth, B. (2000). Expert Judgement and risk perception. Offshore and Polar Engincering Conference, ISOPE, 6, 537-544, Stavanger, June 17-22. http://research.dnv.com/skj/Papers/SkjWen.pdf 